\title{
The Effects of Modified Lymphoscintigraphy Techniques on Sentinel Lymph Node Biopsy Success During the COVID-19 Pandemic Period
}

\author{
CEMIL YÜKSEL ${ }^{1}$, Serdar Çulcu ${ }^{2}$, and Lütfi Doğan ${ }^{2}$ \\ ${ }^{1}$ Affiliation not available \\ ${ }^{2}$ Dr Abdurrahman Yurtaslan Onkoloji Eğitim ve Araştırma Hastanesi
}

July 16, 2020

\begin{abstract}
Background Sampling of the sentinel lymph node (SLN) in breast cancer provides accurate information about the condition of the axilla in $95 \%$ of cases. Technically in detecting SLN; radioactive substance, dyers or both are used. During the COVID-19 (Coronavirus disease 2019) pandemic, delayed images were not taken in lymphoscintigraphy to reduce the risk of transmission by shortening the waiting time in our center. In this study, the effects of early and delayed lymphoscintigraphic images and only early images on our clinical practice were evaluated. Methods We investigated the data of 147 patients in this study who underwent SLNB due to early-stage breast cancer at our institute during the COVID-19 pandemic period (March/April/May 2020) and within 3 months before the pandemic were evaluated. Results Patients were divided into two groups, before pandemic (BP) and pandemic period (PP). BP consisted of patients whose early and delayed images were taken in lymphoscintigraphy whereas PP consisted of those with early images only. There were 74 patients in the BP group and 73 patients in the PP group. Discussion/Conclusions In the present study, we believe that if technically possible, delayed images taken during the lymphoscintigraphy can assist the surgeon in terms of SLN detection and the number of SLNs removed.
\end{abstract}

The Effects of Modified Lymphoscintigraphy Techniques on Sentinel Lymph Node Biopsy Success During the COVID-19 Pandemic Period

\section{Abstract \\ Background}

Sampling of the sentinel lymph node (SLN) in breast cancer provides accurate information about the condition of the axilla in $95 \%$ of cases. Technically in detecting SLN; radioactive substance, dyers or both are used. During the COVID-19 (Coronavirus disease 2019) pandemic, delayed images were not taken in lymphoscintigraphy to reduce the risk of transmission by shortening the waiting time in our center. In this study, the effects of early and delayed lymphoscintigraphic images and only early images on our clinical practice were evaluated.

\section{Methods}

We investigated the data of 147 patients in this study who underwent SLNB due to early-stage breast cancer at our institute during the COVID-19 pandemic period (March/April/May 2020) and within 3 months before the pandemic were evaluated.

\section{Results}


Patients were divided into two groups, before pandemic (BP) and pandemic period (PP). BP consisted of patients whose early and delayed images were taken in lymphoscintigraphy whereas PP consisted of those with early images only. There were 74 patients in the BP group and 73 patients in the PP group.

\section{Discussion/Conclusions}

In the present study, we believe that if technically possible, delayed images taken during the lymphoscintigraphy can assist the surgeon in terms of SLN detection and the number of SLNs removed.

Key words: Breast Cancer; Axillary Lymph Node Dissection; Lymphoscintigraphy; Sentinel Lymph Node; Pandemics

\section{What's Known}

SLNB has replaced ALND in the evaluation of axilla in early-stage breast cancer. Studies have shown that the combined use of preoperative scintigraphic mapping, intraoperative blue dye and gamma probe methods increase the success of SLN detection in breast cancer. Early and delayed images are routinely taken in lymphoscintigraphy but we did not find a study designed like ours evaluating the reflection of the early and delayed images taken during lymphoscintigraphy into clinical practice.

\section{What's New}

During COVID-19 pandemic period, delayed images were not taken in lymphoscintigraphy to reduce the risk of transmission by shortening the waiting time in our center. We examined the effects of taking delayed images on our surgical practice and investigated the importance of delayed images.

\section{Introduction}

Sentinel lymph node biopsy (SLNB) has replaced routine axillary dissection in early-stage breast cancer since the late 1990s [1-3]. Sampling of the sentinel lymph node (SLN) in breast cancer provides accurate information about the condition of the axilla in $95 \%$ of cases [4]. Technically in detecting SLN; radioactive substance, dyers or both are used. Sentinel nodes detected by scintigraphic imaging during the preoperative period can be found with the help of gamma probe and/or after the injection of dyers to the breast during surgery, the stained duct in the axilla and subsequently the dyed lymph node can be observed and surgically removed. There are different applications regarding issues such as the choice of agents to be used (blue dye, radioactive substance or both), the location of injection (periareolar, subareolar, peritumoral), and timing of scintigraphy (morning of surgery or the day before).

During the COVID-19 (Coronavirus disease 2019) pandemic, delayed images were not taken in lymphoscintigraphy to reduce the risk of transmission by shortening the waiting time in our center. In this study, the effects of early and delayed lymphoscintigraphic images and only early images on our clinical practice were evaluated.

\section{Materials and Methods}

In our clinic, to see the drainage from the breast to the axilla routinely on lymphoscintigraphy and to determine the number of increased uptake SLNs, first early then 2 hours later delayed imaging is performed. If the drainage area cannot be detected in the images and no increased uptake SLN is seen, new images are taken after some time if necessary but in the COVID-19 pandemic period it was decided by the surgical and nuclear medicine teams not to take delayed images in order to shorten the waiting time and hence reduce the risk of viral spread and transmission. Our study aimed to investigate the effects of delayed images on SLN biopsy by comparing the two groups. In this study patients who underwent SLNB due to early-stage breast cancer at our institute during the COVID-19 pandemic period (March/April/May 2020) and within 3 months before the pandemic were evaluated. All patients who underwent SLN biopsy were included in the study. Patients with distant metastases, male breast cancer, malignancy other than breast cancer and those who had previously undergone breast surgery or breast surgery without SLNB, as well as patients whose data could not be fully accessed were excluded from the study. After intradermal and 1 intraparenchymal or 
intra/peritumoral injection of $1 \mathrm{mCi}$ of $99 \mathrm{~m}$ Tc labeled nanocolloid (Navidea Biopharmaceuticals, Dublin) to 4 quadrants around the lesion for the purpose of marking the SLN on the morning of the operation or one day before; early (dynamic-SPECT) and 2 hours later delayed (static) images are taken in the Nuclear Medicine unit. The patient is then taken into operation and intraoperative SLNs are searched with a gamma detector. In our clinic we routinely inject $5 \mathrm{cc}$ of methylene blue to each patient. After the patient is intubated, we inject the methylene blue in areola at 4 quadrants and start the operation after 10 minutes of breast massage. Hence, we perform a dual technique SLN biopsy. The patients were taken to surgery after 8 hours of fasting. Patients marked with radioactive substance were injected with a total of $5 \mathrm{cc}$ of methylene blue in areola at 4 quadrants and peritumoral after general anesthesia. After 10 minutes of breast massage, SLN biopsy was started. SLN was accepted as lymph nodes stained in blue or detected by the surgeon during perisentinel dissection in operation or lymph nodes from which high levels of radioactivity was obtained using a portable gamma probe. The removed SLNs were sent for frozen examination. Axillary dissection was not performed in patients who met the criteria specified in the ACOSOG Z0011 study. Final pathologies were obtained in an average of 10 days.

In both groups, number of lesion increased uptake observed in the lymphoscintigraphy, early/delayed increased uptake rate, SLN detection rate, sensitivity, specificity, negative predictive value (NPV), positive predictive value (PPV) of SLNB process, number of positive SLN, axillary dissection, axillary malignant lymph node, age, tumor characteristics ( $\mathrm{T}$ stage, receptor status) and neoadjuvant therapy status were examined in patients. In the study the effect of taking delayed images on lymphoscintigraphy on SLN biopsy success was investigated.

Lymphoscintigraphy reports were examined by scanning electronic files. Micrometastatic lymph nodes were not included in the false negativity rate (FNR). The study protocol was approved by the ethics committee of our institution and the ethics committee approval number is 95/27.05.20.

\section{Statistical Analysis}

SPSS 11.5 software was used in the analysis of the data. For descriptive analysis, quantitative variables were presented as mean \pm standard deviation and median (minimum-maximum), and qualitative variables were presented as number of patients (percentage). Since the normal distribution assumptions were not realized, whether there was a difference between the categories of the qualitative variable, which has two categories in terms of quantitative variable, was analyzed by using the Mann-Whitney $\mathrm{U}$ test. The Chi-squared test and Fisher's exact test were used to evaluate the relationship between two qualitative variables. The statistical significance level was accepted as 0.05 .

\section{Results:}

147 patients were included in our study, and they were divided into two groups, before pandemic (BP) and pandemic period (PP). BP consisted of patients whose early and delayed images were taken in lymphoscintigraphy whereas PP consisted of those with early images only. There were 74 patients in the BP group and 73 patients in the PP group. The mean age of the patients was $52.94 \pm 10.91$. 136 patients (92.5\%) had invasive ductal carcinoma, $5(3.4 \%)$ had invasive lobular carcinoma, 6 (4.1\%) were diagnosed as mixed-type. Estrogen receptor (ER) was positive in 124 patients (84.3\%) and Progesterone receptor (PR) was positive in 117 patients (79.5\%). Cerb-B2 was positive in 33 patients (22.4\%). 12 patients received neoadjuvant therapy.

In lymphoscintigraphy performed in the BP period, early phase increased uptake was not observed in 23 patients, and increased uptake was obtained from 22 of these patients with delayed imaging. Surgery was performed in only 1 patient without increased uptake. In PP, increased uptake was not observed in 12 patients. In BP, no sentinel lymph nodes were found in 2 patients $(2.7 \%)$ during the operation, while in PP, no SLNs were found in 7 patients $(9.5 \%)$ but there was no statistically significant difference between the two groups in terms of SLN detection rate $(\mathrm{p}=0.097)$. Since SLN was not detected in 2 patients in the BP group and 7 in the PP group, axillary dissection had to be performed. Results of SLN and Axillary lymph node dissection (ALND) procedures in both groups are summarized in Figure-1. 
Comparisons of patient characteristics before and during the pandemic are shown in Table 1-2.

While there was a mean of 3.19 SLN in the BP group this figure was shown to be 2.68 in the PP group. The number of metastases in sentinel lymph nodes is higher in the BP period and the difference was statistically significant $(\mathrm{p}=0.04)$. In the BP group, the positive SLN rate per SLN removed was 0.2 , and in the PP group, it was 0.12 .

In 4 of the patients who received neoadjuvant therapy no increased uptake was observed on scintigraphy and 2 of these neither surgically had any positive lymph nodes, and none was found after ALND. While one of the other 2 patients was sentinel-negative, the other one was positive and malignant lymph node was detected when ALND was performed. The increased uptake rate in scintigraphy was $66.6 \%$ in patients who received neoadjuvant therapy.

Of the patients whose SLNB was performed in the pandemic period 55 were negative, 18 were metastatic whereas 4 had false negative results. The sensitivity of SLN biopsies performed in this period was $84.8 \%$ (59.794.8 ) and the specificity was $100 \%$. While the positive predictive value was $100 \%$, the negative predictive value was 92.7 and the accuracy was $94.5 \%$. Of the patients whose SLNB was performed before the pandemic 46 were negative, 28 were metastatic whereas 2 had false negative results. The sensitivity of SLN biopsies performed in this period was $93.1 \%$ (77.2-99.1), and the specificity was $100 \%$. While the positive predictive value was $100 \%$, the negative predictive value was $95.8 \%$ and the accuracy was $97.3 \%$. (Table-3)

As a result of statistical analysis when delayed images were taken by lymphoscintigraphy, increased uptake was observed and information about drainage pathways and SLN numbers were obtained in 12/74 (16.2\%) patients. Although sentinel lymph node detection rate was $97.4 \%$ in BP and $90.4 \%$ in PP, the difference was not statistically significant. It was found that the sensitivity, NPV and accuracy of the SLNB procedure performed after taking delayed images was higher. It was also observed that more SLNs were removed in the SLNB procedure after taking delayed images but the difference did not reach a statistically significant level.

\section{Discussion}

Axillary lymph node status is one of the most important prognostic factors in early breast cancer and is guiding for personalized medicine. Historically, ALND has been used as the most accurate and reliable method to evaluate axillary but ALND has complications such as lymphedema, shoulder dysfunction, nerve injuries that restrict functions and impair quality of life. SLNB is a less invasive method to evaluate lymph node status [5]. The concept of SLNB is based on the idea that tumors are regularly drained through the lymphatic system [6]. Therefore sentinel lymph node (SLN) is the first lymph node where metastasis occurs. SLNB has replaced ALND in the evaluation of axilla in early-stage breast cancer [7].

Over the years, methods such as dyers and radioactive substance injection have been used alone or in combination to detect SLN. Studies have shown that the combined use of preoperative scintigraphic mapping, intraoperative blue dye and gamma probe methods increase the success of SLN detection in breast cancer[8]. However, it is not possible to detect SLN in 1-2\% patients in breast cancer with conventional methods [9]. Techniques applied by each center may differ but overall SLN detection and accuracy rates are over $95 \%$. In our clinical practice we use the combination of radioactive substance and methylene blue injection.

Although there are many studies comparing the methods used in the sentinel lymph node detection in the literature but we did not find a study designed like ours evaluating the reflection of the early and delayed images taken during lymphoscintigraphy into clinical practice. During COVID-19 pandemic period, delayed images were not taken in lymphoscintigraphy to reduce the risk of transmission by shortening the waiting time in our center. In this study, we examined the effects of taking delayed images on our surgical practice. Guidelines for the timing of lymphoscintigraphy are not clear $[1,10]$. Early or delayed image acquisition time is left open-ended in the guidelines and no definite time is specified [1, 11]. NCCN (National Comprehensive Cancer Network) guidelines for breast cancer do not provide detailed information about when to acquire lymphoscintigraphy images and when a delayed image is necessary [2]. In 2014, the German Society of Nuclear Medicine published a guideline on this issue and these instructions refer to the necessity to acquire 
delayed images within 24 hours following an early image[11].

In our clinic, early (dynamic-SPECT) and 2 hours later delayed (static) images are taken following intradermal and 1 intraparenchymal or intra/peritumoral injection of $1 \mathrm{mCi}$ of $99 \mathrm{~m}$ Tc labeled nanocolloid to 4 quadrants around the lesion within 24 hours before the operation.

In a study where Taumberger et al. evaluated early and delayed images, increased uptakes were obtained in delayed images from $52 \%$ of the patients who provided no increased uptakes in early images [12]. In our study, before the pandemic increased uptake were obtained in delayed images in 22 of 23 patients $(95.6 \%)$ who did not provide increased uptakes in early images. Likewise during the pandemic period, in 12 (16.4\%) of the patients whose delayed images were not acquired, increased uptakes could not be obtained.

Single-Photon Emission Computerized Tomography (SPECT) images taken after the radioactive substance is given in lymphoscintigraphy shows axillary drainage pathways and the number of increased uptake lymph nodes $[1,13]$. With the help of the gamma probe, the surgeon will have information about the region where he will search for SLN and the number of SLNs to remove.

The increased uptake duration in lymphoscintigraphy varies according to the location, characteristics and size of the tumor, the patient's neoadjuvant treatment, Body Mass Index (BMI), and the distance between the breast tissue and the axilla [4, 14]. increased uptake is observed in early SPECT images in a group of patients, and not observed in others. Delayed images are preferably taken in patients without fluorescence in early SPECT images. In our center, the delayed image acquisition time is 2 hours after the early image. In this way the aim is to detect in delayed imaging the lymph nodes that cannot be displayed in the early imaging.[15]. During the COVID-19 pandemic, we made SLNB applications by taking only an early SPECT image to reduce the risk of transmission by shortening the waiting time in our center. Therefore, our study is designed to determine how the results of SLNB are affected in patients without delayed lymphoscintigraphy.

To list the main results we obtained in this study; first we found that there were fewer SLNs in patients who underwent SLNB without delayed lymphoscintigraphy (mean $2.68 / 3.19$ ). This difference was not statistically significant $(\mathrm{p}=0.146)$ but the results obtained were due to the limited number of patients and a statistically significant difference could occur if the number of patients was higher. The main reason for this is that we do not know how much SLN increased uptake was to be seen in some of the patients who did not have delayed images. Therefore, as clinicians, we cannot predict a lower limit for the number of LNs we need to remove. For example, we may try to remove 3 SLNs in a patient with 3 increased uptake. If we do not have lymphoscintigraphy and found only 1 SLN, then we end the process. This situation shows us that if there is no delayed image we remove less SLNs and terminate the process. However the most important result is that our rate of SLN detection is lower in patients without delayed imagery. Although there was a ratio of 7 to 2 in our study, no statistically significant difference was found ( $p=0.097$ ). However if this study was conducted with a larger number of patients, we could have found a significant difference. The main parameters that will reveal the reliability and effectiveness of SLNB are the rate of detection, sensitivity, specificity, NPV, PPV and accuracy rates. We have observed that the absence of delayed images reduces the rate of SLN detection. ALND is applied to all patients whose SLN is not detected. As a matter of fact, we had to perform ALND in 7 patients (9.5\%) in the group without delayed imaging, as their SLN was not detected. In 5 of them, there was no axillary metastasis. In the end, this situation led to unnecessary axillary dissection in 5 of 73 patients. In addition, we have seen that our NPV value is lower in our SLN attempts without delayed images in PP. We also observed that our FNR rate is higher in PP with a rate of 4 to 2 . This, in turn reduced the accuracy rate of our SLN procedures made in this period. Although the general results of our procedures performed without acquiring delayed image in PP are within the acceptable limits in the literature, we get better results in the same period when delayed images are acquired. Although acceptable accuracy and detection rates were attained, we have concluded that the figures are better in the procedures performed by taking delayed images. Our suggestion is to perform SLNB by taking the delayed images.

The fact that the rate of metastasis was lower in SLNBs performed in PP was found statistically significant (p $=0.04)$. Neoadjuvant indications and criteria were expanded during the pandemic period. As many patients 
as possible were directed to neoadjuvant therapy in order to postpone their surgical treatments. For this reason it seems probable that a group of patients with high risk of axillary metastasis may have been directed to neoadjuvant therapy during the pandemic period with their surgery being postponed or may have only been given hormonotherapy and followed up. Therefore, we estimate that the rate of SLN metastasis in our patients who underwent surgery during the pandemic period is lower.

In conclusion we believe that if technically possible, delayed images taken during the lymphoscintigraphy can assist the surgeon in terms of SLN detection and the number of SLNs removed.

\section{Statements}

\section{Statement of Ethics:}

In the manuscript, Subjects (or their parents or guardians) have given their written informed consent and that the study protocol was approved by our institute committee on human research. The approval number is $95 / 27.05 .20$.

\section{Conflict of İnterest Statement}

There is no conflict of interest in writing of this article.

\section{Funding Sources}

There is no funding for this study

Availability of data and materials: The datasets used and/or analysed during the current study are available from the corresponding author on reasonable request.

\section{References}

1. Lyman, G.H., et al., Sentinel lymph node biopsy for patients with early-stage breast cancer: American Society of Clinical Oncology clinical practice guideline update. Journal of Clinical Oncology, 2016.

2. Network, N.C.C., Clinical Practice Guidelines in Oncology-Breast Cancer Version 2. http://www. nccn. com, 2011.

3. Zurrida, S. and U. Veronesi, Milestones in breast cancer treatment. The breast journal, 2015. 21 (1): p. $3-12$.

4. Kauffmann, G.W., E. Moser, and R. Sauer, Radiologie: mit 115 Tabellen; Fragen und Antworten . 2006: Elsevier, Urban\&FischerVerlag.

5. Krag, D.N., et al., Surgical resection and radiolocalization of the sentinel lymph node in breast cancer using a gamma probe. Surgical oncology, 1993. 2 (6): p. 335-340.

6. Reintgen, D., et al., The orderly progression of melanoma nodal metastases. Annals of surgery, 1994. 220 (6): p. 759 .

7. Veronesi, U., et al., A randomized comparison of sentinel-node biopsy with routine axillary dissection in breast cancer. New England Journal of Medicine, 2003. 349 (6): p. 546-553.

8. Nielsen, K.R., et al., The diagnostic value of adding dynamic scintigraphy to standard delayed planar imaging for sentinel node identification in melanoma patients. European journal of nuclear medicine and molecular imaging, 2011. 38 (11): p. 1999.

9. van der Ploeg, I.M., et al., The yield of SPECT/CT for anatomical lymphatic mapping in patients with melanoma. Annals of surgical oncology, 2009. 16 (6): p. 1537-1542.

10. Giammarile, F., et al., The EANM and SNMMI practice guideline for lymphoscintigraphy and sentinel node localization in breast cancer.European journal of nuclear medicine and molecular imaging, 2013.40 (12): p. $1932-1947$. 
11. Schmidt, M., et al., DGN-Handlungsempfehlung (S1-Leitlinie) Verfahrensanweisung für die technische Durchführung der nuklearmedizinischen Wächter-Lymphknoten-Diagnostik . 2014, Stand 10/2014-AWMFRegisternummer 031-033 [accessed 2018 Nov 9]. Available ....

12. Kim, T., A.E. Giuliano, and G.H. Lyman, Lymphatic mapping and sentinel lymph node biopsy in earlystage breast carcinoma: a metaanalysis. Cancer, 2006. 106 (1): p. 4-16.

13. Dimcheva, M., S. Sergieva, and B. Robev, PRACTICAL METHODOLOGY FOR LYMPHOSCINTIGRAPHY AND SENTINEL LYMPH NODE BIOPSY IN BREAST CANCER.Journal of Nuclear Medicine, 2020. 61 (supplement 1): p. 3027-3027.

14. Sadeghi, R. and L. Zarifmahmoudi, Application of Lymphatic Mapping and Sentinel Node Biopsy in Surgical Oncology, in Clinical Nuclear Medicine . 2020, Springer. p. 431-458.

15. Kuemmel, S., et al., Prospective, multicenter, randomized phase III trial evaluating the impact of lymphoscintigraphy as part of sentinel node biopsy in early breast cancer: SenSzi (GBG80) trial.Journal of Clinical Oncology, 2019. 37 (17): p. 1490-1498.

Table 1. Patient characteristics

\begin{tabular}{|c|c|c|c|c|}
\hline Variables & Variables & Variables & COVID-19 Status & $\mathrm{CO}$ \\
\hline & & & $\begin{array}{l}\text { Before COVID-19 } \\
\mathrm{N}\end{array}$ & $\begin{array}{l}\mathrm{Be} \\
\%\end{array}$ \\
\hline \multirow[t]{3}{*}{ Diagnosis } & Invasive ductal carcinoma & 70 & 70 & 94. \\
\hline & Invasive lobular carcinoma & 3 & 3 & 4. \\
\hline & Mixed & 1 & 1 & 1. \\
\hline \multirow[t]{2}{*}{ ER } & Negative & 11 & 11 & \\
\hline & Positive & 63 & 63 & \\
\hline \multirow[t]{2}{*}{ PR } & Negative & 15 & 15 & \\
\hline & Positive & 59 & 59 & \\
\hline \multirow[t]{2}{*}{ HER-2 } & Negative & 58 & 58 & \\
\hline & Positive & 16 & 16 & 2 \\
\hline \multirow{2}{*}{ Neoadjuvant Therapy } & No & 68 & 68 & \\
\hline & Yes & 6 & 6 & 8. \\
\hline \multirow[t]{2}{*}{ İncreased uptake in Lymphoscintigraphy } & Not Observed & 1 & 1 & \\
\hline & Observed & 73 & 73 & \\
\hline \multirow[t]{2}{*}{ Axillary dissection } & Not performed & 47 & 47 & \\
\hline & Performed & 27 & 27 & \\
\hline \multirow[t]{3}{*}{ T Stage } & 1 & 30 & 30 & \\
\hline & 2 & 37 & 37 & \\
\hline & 3 & 2 & 2 & \\
\hline \multirow[t]{2}{*}{ Sentinel Lymph Node Detection Status } & Not found & 2 & 2 & 2. \\
\hline & Found & 72 & 72 & \\
\hline
\end{tabular}

a: Chi-squared test, b: Fisher's exact test

Table 2. Patient characteristics and lymph node status

\begin{tabular}{lll}
\hline Variables & COVID-19 Status & COVID-19 Status \\
\hline & Before COVID-19 & COVID-19 Period \\
Age & Mean \pm SD & Mean \pm SD \\
Number of Sentinel Lymph Node Removed & $\mathbf{5 2 . 0 1} \pm 10.82$ & $\mathbf{5 3 . 8 8} \pm 10.99$ \\
& $3.19 \pm 2.11$ & $\mathbf{2 . 6 8} \pm 2.01$
\end{tabular}




\begin{tabular}{lll}
\hline Variables & COVID-19 Status & COVID-19 Status \\
\hline Mean Number of Metastases in Sentinel Lymph Nodes & $\mathbf{0 . 6 5} \pm \mathbf{1 . 1 2}$ & $\mathbf{0 . 3 4} \pm \mathbf{0 . 7 9}$ \\
Mean Number of Lymph Nodes Removed at Axillary Dissection & $\mathbf{1 6 . 5 6} \pm 4.98$ & $\mathbf{1 5 . 8 4} \pm 6.96$ \\
Mean Number of Axillary Metastatic Lymph Nodes & $\mathbf{1 . 1 1} \pm \mathbf{1 . 9 3}$ & $\mathbf{2 . 3 7} \pm \mathbf{3 . 4 4}$ \\
\hline
\end{tabular}

SD: Standard Deviation

Table 3. Method efficacy comparison

\begin{tabular}{lll}
\hline & Before COVID-19 & COVID-19 Period \\
\hline & $\%$ & $\%$ \\
Sensitivity & $93.1(77.2-99.1)$ & $84.8(59.7-94.8)$ \\
Specificity & 100 & 100 \\
Negative Predictive Value & 95.8 & 92.7 \\
Positive Predictive Value & 100 & 100 \\
Accuracy & 97.3 & 94.5 \\
\hline
\end{tabular}

Legends:

Figure 1. Patients flow chart

List of abbreviations

Sentinel Lymph Node Biopsy: SLNB

Sentinel Lymph Node: SLN

Coronavirus Disease 2019: COVID-19

Single-Photon Emission Computerized Tomography: SPECT

Negative Predictive Value: NPV

Positive Predictive Value: PPV

False Negativity Rate: FNR

Before Pandemic: BP

Pandemic Period: PP

Estrogen Receptor: ER

Progesterone Receptor: PR

Axillary Lymph Node Dissection: ALND

Non-Sentinel Metastatic Lymph Node: NSMLN

Standard Deviation: SD

National Comprehensive Cancer Network: NCCN

Body Mass Index: BMI 


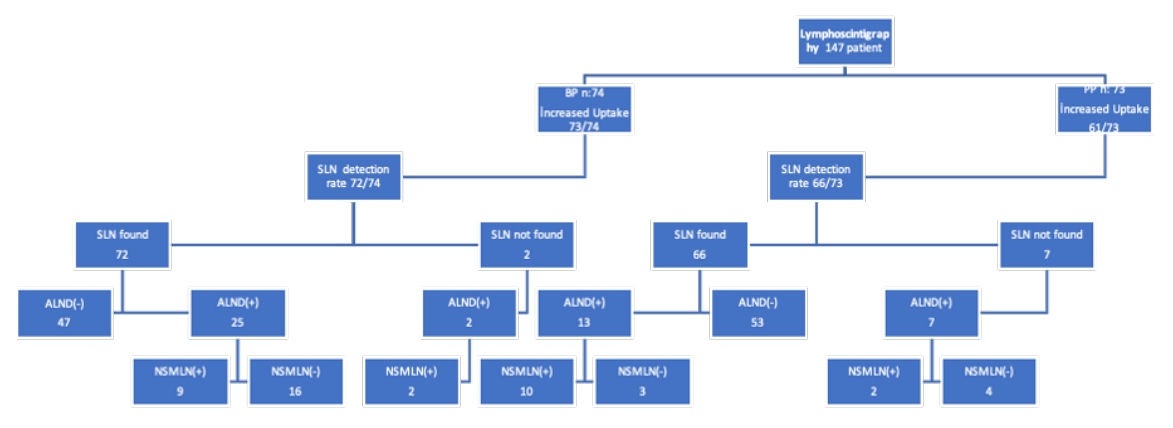

